\title{
Role of Serum Cholesterol Level in Pathogenesis of OCD
}

\section{Madhura TK*}

Deapartment of Biochemistry, Defense Institute of Advanced Technology, Near Khadakwasla Dam, Girinagar, Pune 411025, India

\begin{abstract}
Etiology and pathophysiology of obsessive compulsive disorder remains inconclusive till today. Multiple converging lines of evidence suggest that neurobiology plays a significant role in the etiology of obsessive compulsive disorder (OCD). Certain brain areas frequently involved in symptomatology of OCD, analyzed by neuroimaging studies. The strongest pharmacologic evidence concerns the serotonergic system and the well-established efficacy of potent serotonin reuptake inhibitors in the treatment of OCD. Cholesterol forming the vital component of neurons regulates neurochemical activity. Reduced serum cholesterol level causes hyperactivity of serotonin reuptake receptor activity precipitating symptoms of OCD.
\end{abstract}

Keywords: OCD; Serotonin; Receptors; Neurotransmitters; Cholesterol

\section{Introduction}

Obsessions are recurrent, persistent, thoughts, images or impulses that are experienced as intrusive \& inappropriate [1].

Compulsions are repetitive behaviors or mental acts that the person feels driven to perform in response to an obsession or according to rigid rules [1].

Obsessive -compulsive disorder is the fourth commonest psychiatric disorder with disability in severe cases often comparable to the disability associated with mental illness such as schizophrenia and bipolar disorder. $2 \%$ of adults suffer from this disorder $[2,3]$

The OCD usually presents at the age of 20-30 yr. If it presents in older age (in 4th decade) it usually comes after a stressful situation in life. OCD usually begins in adolescence or early adulthood although it can begin in childhood. Nearly $65 \%$ of the patients have the onset before 25 years of age and $<15 \%$ have onset after 35 years of age $[2,3]$.

Epidemiological studies show that around 2\% of adults suffer from this disorder.

\section{Etiology $[3,4]$}

\section{Neurobiological factors}

Onset of OCD symptoms follow neurological injury secondary to infection, head injury or neuronal over activity.

Efficacy of serotonin reuptake inhibitors in symptomatic improvement in OCD cases has proved role of seroronin in pathophysiology of OCD.

\section{Neurophysiological factors}

Positron emission tomography (PET) studies suggest increased glucose metabolism in orbital frontal cortex, pre-frontal cortex and anterior cingulate gyrus, right caudate nucleus.

\section{Neuroanatomical factors}

Neuroimaging studies that have been implicated the frontal-basal ganglio thalamic cortical circuit in the pathogenesis of the disorder

\section{Neurochemical basis}

Oxidative stress causes neuronal damage in the above mentioned areas. Damage caused disturbs intracellular events resulting in impaired release of neurotransmitters like serotonin, dopamine which have been proposed in the pathophysiological basis of OCD, thus decreasing the synaptic levels of neurotransmitters leading to symptomatology.

\section{Neuropsychological factors}

\section{Immunological factors}

Subgroup of youngster's present OCD symptoms triggered or symptoms get precipitated with group a beta hemolytic streptococcal infection

\section{Family history}

$50 \%$ childhood cases are familial.

\section{Genetic factors}

Studies suggest that $30 \%$ of adults with OCD had immediate family member with symptoms of OCD. Research suggests that if one parent has OCD the chances of the child having it are between $2 \%$ and $8 \%$.

\section{Behavioral factors}

According to Hobart Mowrer's conditioning theory, previously neutral stimulus becomes aversive, as a result of being associated with an unrelated aversive stimulus

Compulsions become strengthened as a result of anxiety reducing properties.

\section{Types of OCD}

There are five main types of OCD that commonly appear in sufferers [6,7].

\section{Washers and cleaners}

Contamination obsessions are the most common type of obsessions in OCD. They are typically characterized by a fear of dirt or germs.

*Corresponding author: Madhura TK, MBBS, MD (Biochemistry), Defense Institute of Advanced Technology, Near Khadakwasla dam, Girinagar, Pune 411025, India, Tel: 9921041635 E-mail: madhuratksharma@gmail.com

Received: May 15, 2015; Accepted: May 20, 2015; Published May 22, 2015

Citation: Madhura TK (2015) Role of Serum Cholesterol Level in Pathogenesis of OCD Biochem Anal Biochem 4: 183. doi:10.4172/2161-1009.1000183

Copyright: ( 2015 Madhura TK. This is an open-access article distributed unde the terms of the Creative Commons Attribution License, which permits unrestricted use, distribution, and reproduction in any medium, provided the original author and source are credited. 
Obsession: a constant fear of contamination and being infected with disease and infecting others. Compulsion: sufferer avoids or removes all possible sources of germs and dirt. Washers may repeatedly wash their hands or take showers while cleaners may clean their house repeatedly.

The cleaning compulsions include excessive or ritualized hand washing, showering, bathing, tooth brushing, and elaborate toilet routines.

\section{Checkers}

Obsession: a fear of forgetting to lock or switch something off will cause harm for which they will be blamed. Compulsion: time consuming rituals that involve checking \& re-checking the objects of their obsession for eg. Locks/ swithces.

Patients with doubts typically worry that something terrible may happen because they have not completed an act thoroughly or completely. Such patients can spend hours checking before leaving their home.

\section{Orderers}

Obsession: To organize or arrange objects in a specific pattern or order before performing a daily task.

Compulsion: Arranging things in a certain 'correct' way. If someone else touches or moves those objects out of place, they will feel extremely upset.

\section{Hoarders}

Obsession: Excessive fear of discarding certain objects in the belief that they may be of use in the future.

Compulsion: Keeping or collecting items those are often useless. Even if some are useful, hoarders tend to collect so many items that they are unable to finish using all of them.

\section{Sexual and aggressive obsessions}

Patients with these obsessions are plagued by thoughts, images or urges that are sexual or aggressive in nature. Patients often fear that they may commit a sexually unacceptable act such as touching the private parts of immediate family members. Aggressive obsessions include fears that one might harm self or others on an impulse (fear of stabbing with a knife, jumping in front of a car, leaping out of an open window) or because not careful enough (fear of hitting a pedestrian because of not being careful), and fear of blurting out obscenities or insults $[5,6]$.

\section{Neurotransmitterinvolvedin OCD pathophysiology}

Serotonin is a monoamine neurotransmitter, biochemically derived from tryptophan. Primarily found in gastrointestinal tract, Central Nervous System, platelets etc. Functions of serotonin include the regulation of mood, appetite, sleep, cognitive functions, including memory and learning $[8,9]$. Serotonin is the major neurotransmitter in raphe nuclei, which are grouped into nine pairs and distributed along the brainstem, centered around the reticular formation. Axons of neurons in the "caudal" raphe nuclei terminate at deep cerebellar nuclei, cerebellar cortex , spinal cord. Serotonin is released at terminal synaptic boutons acting at a wide gap of $>20 \mu \mathrm{m}$ synapse and acts to activate 5-HT receptors located on the dendrites, cell bodies and presynaptic terminals of adjacent neurons. Serotonin receptors are G protein coupled seven transmembrane (or "heptahelical") receptors that activate an intracellular second messenger cascade except 5- $\mathrm{HT}_{3}$, which is liagand gated ion channel [10].
SERT spans the plasma membrane 12 times. It belongs to NE, DA, SERT monoamine transporter family. Transporters are important sites for agents that treat psychiatric disorders. Both drugs that reduce the binding of serotonin to transporters (selective serotonin reuptake inhibitors, or SSRIs) and, less often, that increase it (selective serotonin reuptake enhancers, or SSREs) are used to treat mental disorders. About half of patients with OCD are treated with SSRIs. Fluoxetine is an example of a selective serotonin reuptake inhibitor, and tianeptine is an example of a selective serotonin reuptake enhancer. The highly evolutionarily conserved serotonin transporter (SERT) regulates the entire serotonergic system and its receptors via modulation of extracellular fluid serotonin concentrations [11].

\section{Cholesterol and OCD}

Cholesterol is the vital component of eukaryotic cell membranes and plays a crucial role maintaining cellular function, membrane dynamics, transport across cell membranes and physical protection. The alteration in membrane physical properties is due to interaction of cholesterol moiety with varies protein components in cellular membranes.

The heterogeneity in cholesterol distribution in membrane domains sequester proteins contributing to significance membrane function. The seven transmembrane domain G-protein coupled receptors (GPCRs) are among the largest protein families in mammals and represent $2 \%$ of the total proteins coded by the human genome. They mediate transduction events, which are the primary means of cellular communication and response to external environment. The recently reported role of cholesterol in the function and organization of the neuronal serotonin 1A receptor, a representative of the GPCR family which is present endogenously in the hippocampal region of the brain, is highlighted. The neurotransmitter release at the synapses underlies fundamental brain activities such as cognition, emotion, and memory. It is therefore natural to speculate that the neurotransmitter release depends on the cholesterol level in the brain. A low cholesterol level may alter the pattern of the release, which in turn perturbs the brain activities to the extent that it could lead to behavioral dysfunction, depression, suicide, and memory loss [12].

\section{Cholestrol in pre-synaptic terminals}

There exists an elaborate assembly of protein and lipid components, releasing the transmitter from pre-synaptic neurons. Cholesterol is the vital component of exocytosis apparatus playing a crucial role in biogenesis and transport of neurotransmitters and other substances in synaptic vesicles.

Synaptosomal preparations are used to to calculate the lipid composition of pre synaptic terminals. The cholesterol: phospholipid ration lies between 0.5: 1 in plasma membranes. The inner leaflet of synaptic membranes contains eight-fold more cholesterol than its outer counterpart and that this distribution is affected by disease and aging. Syntaxin and SNAP25, which form the soluble N-ethylmaleimidesensitive factor attachment protein receptor (SNARE) complex with vesicle-associated membrane protein (VAMP), but none of the other exocytosis relevant proteins tested partitioned to the detergentinsoluble cholesterol-rich membrane fraction [13-15].

Presynaptic transmitter release involves interplay of two highly specialized compartments, the membrane at the docking zone and the membrane of synaptic vesicles. The molecular composition of synaptic vesicles has been characterized, since these organelles can be purified to a high degree from synaptosomes due to their abundance and uniform size. Cholesterol has profound effects on the biophysical 
properties of membranes, thereby affecting the function of membraneresident signaling components including ion channels, transporters and receptors. In a molecular level, cholesterol can affect synaptic membrane fusion in a number of different ways:

cholesterol is proposed to form laterally separated membrane domains called rafts, in cooperation with another brain lipid sphingolipid. The rafts could play a role in driving 58 the clustering of SNAREs into a specific membrane site, which may be necessary for productive fusion.

it is shown that cholesterol induces the clustering of the SNARE proteins by promoting self-assembly of the proteins without the rafts. The clustering of SNAREs is thought to be helpful for docking and fusion of vesicles.

due to its unique inverse wedge-like molecular shape, cholesterol is thought to play a role in membrane fusion which involved curving and bending of the bilayers [16].

\section{Estimation of Serum Cholesterol level}

\section{Method: cholesterol oxidase peroxidase methodology (Agappe diagnostics)}

Sample collection: After taking informed consent, under thorough aseptic precautions, $2 \mathrm{ml}$ of venous sample is collected from the patient in a plain vacutainer and allowed to clot. After one hour, the vacuitainer is centrifuged at $2500 \mathrm{rpm}$ for $5 \mathrm{~min}$ and serum is exteracted and stored.

\section{Principle of CHOD-POD method}

Enzymatic colorimetric determination of total cholesterol according to the following reactions.

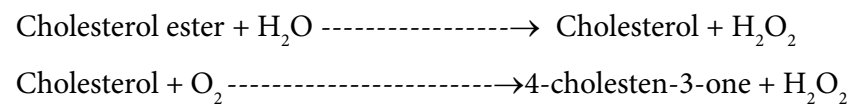

$2 \mathrm{H}_{2} \mathrm{O}_{2}+$ Phenol +4 - aminoantipyrene ------ $\rightarrow$ red quinine $+4 \mathrm{H}_{2} \mathrm{O}$

\section{Reagent composition}

\section{Cholesterol Standard: $1 \times 5$ ML (Table 1)}

Preparation and stability of working reagent: Dissolve contents of reagent $2\left(R_{2}\right)$ with the amount of reagent $1\left(R_{1}\right)$ indicated on the vial label. The working reagent is stable for 90 days at $2-8^{\circ} \mathrm{C}$.

Wavelength for estimation: $505-630 \mathrm{~nm}$

Linearity: $500 \mathrm{mg} / \mathrm{dL}$

\section{Procedure}

\begin{tabular}{|c|c|c|c|}
\hline & Blank & Standard & Sample \\
\hline Working reagent & $1000 \mu \mathrm{L}$ & $1000 \mu \mathrm{L}$ & $1000 \mu \mathrm{L}$ \\
\hline Standard & - & $10 \mu \mathrm{L}$ & - \\
\hline Sample & - & - & $10 \mu \mathrm{L}$ \\
\hline
\end{tabular}

Mix and incubate for $5 \mathrm{~min}$. at $37^{\circ} \mathrm{C}$. Measure the absorbance of sample and standard against reagent blank.

\begin{tabular}{|l|c|l|c|}
\hline Cholesterol $\mathrm{R}_{1}: 2 \times 50 \mathrm{ml}$ & Cholesterol $\mathrm{R}_{2}: 2 \times 50 \mathrm{ml}$ \\
\hline Pipes buffer $(\mathrm{pH} 6.9)$ & $50 \mathrm{mmol} / \mathrm{L}$ & Cholesterol esterase & $\geq 200 \mathrm{U} / \mathrm{L}$ \\
\hline Phenol & $24 \mathrm{mmol} / \mathrm{L}$ & Cholesterol oxidase & $\geq 250 \mathrm{U} / \mathrm{L}$ \\
\hline Sodium cholate & $0.5 \mathrm{mmol} / \mathrm{L}$ & Peroxidase & $\geq 1000 \mathrm{U} / \mathrm{L}$ \\
\hline & & 4-aminoantipyrene & $0.5 \mathrm{mmol} / \mathrm{L}$ \\
\hline
\end{tabular}

Table1: Cholesterol standard of concentration: $200 \mathrm{mg} / \mathrm{dl}$.

\begin{tabular}{|c|c|c|}
\hline & Cases & Controls \\
\hline Mean age \pm SD (years) & $34.5 \pm 9.26$ & $34.03 \pm 8.52$ \\
\hline Sex & $16 \mathrm{M} / 14 \mathrm{~F}$ & $17 \mathrm{M} / 13 \mathrm{~F}$ \\
\hline \multicolumn{2}{|c|}{ Table 2: Baseline characteristics of patients. }
\end{tabular}

\section{Calculation}

Cholesterol concentration $(\mathrm{mg} / \mathrm{dL})=($ Absorbance of sample / Absorbance of standard) $\times 200$

Normal Range: $140-200 \mathrm{mg} / \mathrm{dLs}$

\section{Method}

This is a case control study on 30 cases and 30 control group with match of age, sex and inclusion /exclusion criteria. Selected age group is between 15-45 years. excluding the cases and controls with history of metabolic disorders, smoking, alcohol consumption, unwillingness in participation in study.

\section{Results}

All the calculations were done using Microsoft Office Excel 2010 and statistical analysis was done using the Graph Pad Prism software, version 5.01 .

The collected data was analyzed by applying paired t test. P-value less than $0.05(\mathrm{P}<0.05)$ was considered to be statistically significant (S). $\mathrm{P}$-value less than $0.001(\mathrm{P}<0.001)$ was considered to be statistically highly significant (HS). P-value more than $0.05(\mathrm{P}>0.05)$ was considered to be statistically non-significant (NS) (Table 2).

The average age of subjects in controls is 34.03 years and 34.5 years in patients with OCD, which shows that mean age is nearly equal in controls and cases.

Sex distribution in controls 17 males and 13 females and in patients with OCD 16 males and 14 females, which shows that sex ratio is nearly equal in controls and cases (Graph 1).

There is significant decrease in serum cholesterol in OCD cases as compared to controls since ( $\mathrm{p}$ value 0.0047 i.e $<0.05)$ (Graph 2).

\section{Discussion and Conclusion}

Obsessive-compulsive disorder (OCD) is a neuropsychiatric disorder characterized by recurrent distressing thoughts and repetitive behaviors or mental rituals performed to reduce anxiety. Symptoms are often accompanied by feelings of shame and secrecy because patients realize the thoughts and behaviors are excessive or unreasonable. This secrecy, along with a lack of recognition of OCD symptoms by health care professionals, often leads to a long delay in diagnosis and treatment

Brain is highly in enriched in cholesterol containing almost one quarter of total bodily cholesterol. In neurons, cholesterol makes up nearly $40 \%$ of total membrane lipids and is in equilibrium with serum cholesterol level. Lowered serum cholesterol levels as seen in cases with respect to controls causes reduced levels of neuronal membrane cholesterol, which cause impaired release of serotonin due to inefficient exocytosis and SERT dysfunction causing exaggerated uptake of serotonin from synapse. Thus there exists relative deficiency of serotonin in the synapses, which impairs neurotransmission leading to misfiring of neurons and misconveyed impulse transmission precipitating symptoms of OCD [9].

\section{OCD and serum cholesterol level}

Cholesterol is an essential component of eukaryotic membranes 


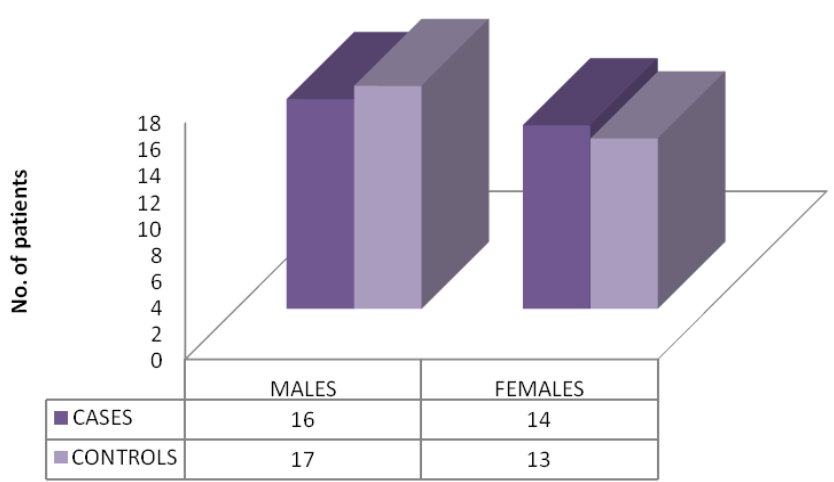

Graph 1: Bar Diagram Showing Sex Wise Distribution In Cases And Controls

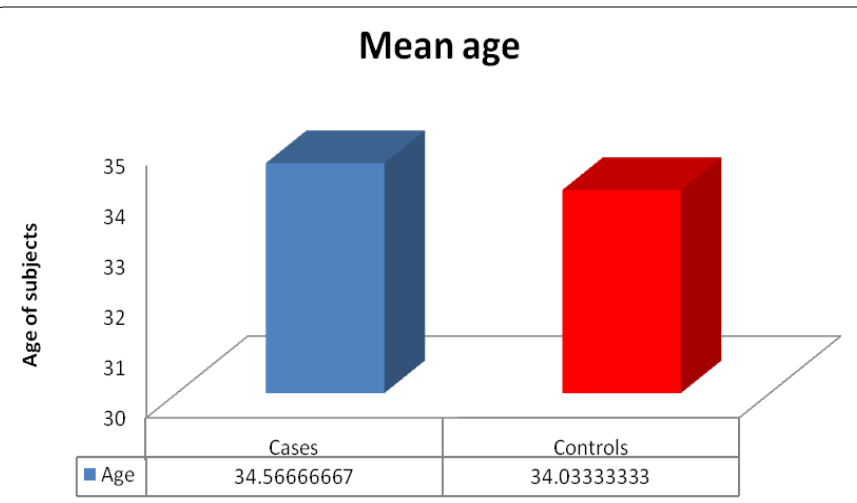

Graph 2: Bar Diagram Showing Mean Age Distribution between Cases and Controls.

\begin{tabular}{|c|c|l|l|}
\hline Group & S. cholesterol level $(\mathbf{m g} / \mathbf{d L})$ Mean \pm SD & T value & p value \\
\hline Cases & $149.33+25.65$ & 4.88 & $<0.05$ \\
\hline Controls & $164.5+16.95$ & & \\
\hline
\end{tabular}

Table 3: Comparison of serum cholesterol level between cases and controls.

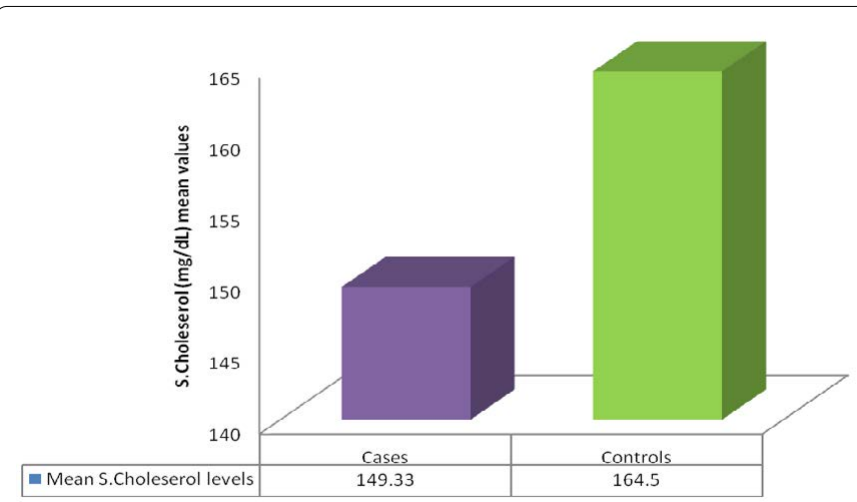

Graph 3: Bar Diagram Showing Comparison of Mean Value Serum Cholesterol Level Between Cases and Controls.

and plays a crucial role in membrane organization, dynamics and function. Cholesterol is a multifaceted molecule, which serves as essential membrane component, as cofactor for signaling molecules and as precursor for steroid hormones. Cholesterol plays a vital role in the acitivity of membrane transporter e.g SERT (serotonin transporter).

$>$ Reduced Serum cholesterol levels in OCD patients also depict low levels of neuronal membrane cholesterol and the increased activity of serotonin transporter (SERT), integral membrane protein which reuptakes serotonin from synapse leading to relative deficiency of serotonin in the synapse that inturn lead to symptoms of OCD.

$>$ Lowered serum cholesterol levels causes impaired release of neurotransmitter serotonin because of inefficient exocytosis of presynaptic neuronal vesicles. And SERT activity is impaired resulting in faster and exaggerated serotonin uptake. Thus the synapse becomes deficient in serotonin causing neuronal irritability and excessive transmission of impulses resulting in OCD symptoms.

$>$ In the present stud $y$, there was significant decrease in serum cholesterol levels in cases $(149.33 \pm 25.65)(\mathrm{p}<0.05)$ (Table 3 and Graph 3) as compared to the control group $(164.5 \pm 16.95)(\mathrm{p}>0.05)$. This shows that the role of decreased serum cholesterol level in the pathogenesis of OCD

\section{Acknowlegement}

Am grateful to our beloved and well known Psychiatrist Dr Vasudeo Parlikar HOD Dept of Psychiatry KEM Hospital and Dr Ajit P Bhale, Associate Professor BJ Govt Medical college Pune-01 for their precious guidance and support.

\section{References}

1. Sadock BJ, Sadock VA, Ruiz P (2009) Chapter 14: Anxiety disorders: Obsessive compulsive disorder. Kaplan and Sadock's Comprehensive Textbook of Psychiatry (9th edn), Lippincot William and Walkins 2: 1849-1851.

2. Fenske JN, Schwenk TL (2009) Obsessive compulsive disorder: diagnosis and management. Am Fam Physician 80: 239-245.

3. Subramaniam M, Abdin E, Vaingankar JA, Chong SA (2012) Obsessive-compulsive disorder: prevalence, correlates, help-seeking and quality of life in a multiracial Asian population. Social Psychiatry Psychiatric Epidemiology 47: 2035-43.

4. Khanna S (1999) Obsessive Compulsive Disorder. In Textbook of Postgraduate Psychiatry, Jaypee Publishers, New Delhi. 1: 262-274

5. Pauls DL, Mundo E, Kennedy JL (2002) Text book of Neuropharmacology. Chapter 112: The Pathophysiology and Genetics of OCD .Charles Nemeroff. American College of Neuropsychopharmacology 1639-1661.

6. Sadock BJ, Sadock VA, Ruiz P (2009) Kaplan and Sadock's Comprehensive Textbook of Psychiatry. Chapter 14: Anxiety disorders: Obsessive compulsive disorder (9th edn), Lippincot William and Walkins 2: 1849-1851.

7. Ahuja NA short textbook of psychiatry. Chapter 8: Neurotic, stress related and Somatoform disorders (7th edn), Jaypee Brothers, Medical Publishers 89-112.

8. Ganong WF Review of medical Physiology. Chapter 7: Neurotransmitters and neuromodulators (23rd edn), Tata Mc Graw Hill education private limited. 129-148.

9. Eisenhofer G, Whitley RJ, Rosano TG Tietz Textbook of clinical chemistry and molecular diagnostics. Chapter 30: Catecholamines and serotonin. Burtis CA, Ashwood ER, Bruns DE (5th edn), Elsevier 851-894.

10. Svenningsson $P$, Chergui K, Rachleff I, Flajolet M, Zhang X, et al. (2006) Alterations in 5-HT1B receptor function by $\mathrm{p} 11$ in depression-like states. Science 311: 77-80.

11. Murphy DL, Lesch KP (2008) Targeting the murine serotonin transporter: insights into human neurobiology. Nature Reviews Neuroscience 9: 85-96.

12. Zimmermann H (1982) Biochemistry of the isolated cholinergic vesicle Neurotransmitter Vesicles, Academic Press, London. 271-304.

13. Nägler K, Mauch DH, Pfrieger FW (2001) Glia-derived signals induce synapse formation in neurones of the rat central nervous system. J Physiol 533: 665-679.

14. Kunugi H, Takei N, Aoki H, Nanko S (1997) Low serum cholesterol in suicide attempters. Biol Psychiatry 41: 196-200.

15. Jablonska RJ, Poprawska I (2000) Levels of serum total cholesterol and LDLcholesterol in patients with major depression in acute period and remission. Medical science monitor: international medical journal of experimental and clinical research 6: 539-47.

16. Gönenç A, Tokgöz D, Aslan S, Torun M (2005) Oxidative stress in relation to lipid profiles in different stages of breast cancer. Indian J Biochem Biophys 42: 190-194. 